\title{
An Experimental Study of EMG Signal Features for Motion Discriminations Using Support Vector Machine
}

\author{
Takuya Shinozaki $^{a}$, Kazuki Kojima, Satoshi Ito ${ }^{\mathrm{b}}$ and Minoru Sasaki ${ }^{\mathrm{c}}$ \\ Dept. of Human and Information Systems, Faculty of Engineering \\ Gifu University Gifu, Japan 1-1 Yanagido, Gifu, 501-1193 \\ ap3128015@edu.gifu-u.ac.jp, bsatoshi@gifu-u.ac.jp(corresponding author), 'sasaki@gifu-u.ac.jp
}

Keywords: Surface EMG, Signal processing, Support Vector Machine.

\begin{abstract}
This paper treats a discrimination problem of wrist/hand motion patterns from EMG signal. We examined which of the following signal features was appropriate: raw signal, integrated signal (IEMG), the max frequency component, power spectrum or rising voltage level. For the discrimination algorism, a Support Vector Machine (SVM) was introduced. As a result, around 80\% discrimination rate was accomplished from integrated signal, power spectrum and rising voltage level. The IEMG signal scored the highest $83.3 \%$ discrimination rate.
\end{abstract}

\section{Introduction}

The electromyographic (EMG) signal is considered to be applicable for the control of prosthetic arms and legs [1][2]. It has many advantages: Surface-EMGs are non-invasively measurable by attaching electrodes to a person's skin. Moreover, exerted force information can be estimated from EMG signals. This makes the EMG signal a potential candidate for the control of prosthetics.

A surface-EMG consists of a set of complex signals from many muscle fibers around the electrode and is affected by many different muscle fibers [2]. Thus the action of an individual muscle from the surface-EMG is difficult to discriminate. Furthermore, the voltage level of an EMG signal is very low and so easy to be effected from noise. To solve these problems, EMG signals and their application have been studied from many aspects [3][4].

This study aims at establishing a new human interface using some forearm EMG signals so as to control mainly robotic devices, especially a hand rehabilitation support system we are developing [7]. This paper investigates which of the following signal features is more effective to discriminate finger and wrist motions: raw signal, integrated signal, the max frequency component, power spectrum, or rising voltage level, As an algorithm to discriminate the hand motion, we adopt the support vector machine because of its high classification performance.

\section{Purpose}

EMG signals would contain efficient information for the human motion, because it affects directly to human motion actuators, i.e., muscles. However, the raw signal contains noises as well as different components from the target muscle, and its direct, i.e., non-processed, usage to the discrimination requires much computational resources due to its high-dimensionality. Therefore, some pre-processing should be introduced before being utilized for motion discrimination.

The hand rehabilitation system we have been developing [7] requires the detection of the following eight motions to assist the hand motion: pronation/supination and dorsal/volar flexion of the wrist, thumb extension/flexion and four fingers extension/flexion. These motions are generated mainly from the following eight muscles: pronator teres, spinatior, extensor/flexor carpi ulnaris, flexor/abductor pollicis longus, flexor digitorum superficialis and extensor digitorum. Thus we tried to discriminate motions by attaching the electrodes around these muscles. 
The purpose of this paper is to investigate what kind of EMG signal features, obtained from the electrodes targeting to the above eight muscles and then be pre-processed, is effective to discriminate the above eight motions. As the feature of the signals, we choose the following five, integrated signal, the max frequency component, power spectrum and rising voltage level: how these features are obtained is briefly explained below. The sampling rate of our EMG measurement system was $3 \mathrm{kHz}$.

I ) Raw data (4800[dimension])

We detect the time $t_{\max }$ when the total sum of all channel voltages is maximized. Then, the EMG signals $0.1 \mathrm{sec}\left(300\right.$ [sample]) before and after $t_{\max }$ are extracted, and normalized by the absolute value of its maximum voltage in each subject. Data dimension is 600 [samples] $* 8[\mathrm{ch}]=4800$ [dimensions]. This raw data is examined for comparison of other signals.

II ) IEMG (4800[dimension])

IEMG is an integration of raw data that are four times amplified after low-pass filtered with $4.8 \mathrm{~Hz}$ cutoff frequency. They are automatically obtained from our EMG measurement systems. Afterwards, the same operation as I) is applied. Data dimension is 600 [sample] $* 8[\mathrm{ch}]=4800$ [dimension].

III) Power spectrum (4096[dimension])

The 256 samples before and after $t_{\max }$ are extracted from each channel. The frequency component in this time range is used as a signal feature, i.e., their power spectrum is calculated and used as the data for discrimination. Data dimension is 512 [sample] $* 8$ [ch] $=4096$ [dimension].

IV) FFT peak (8[dimension])

The largest component of power spectrum in each $8 \mathrm{ch}$ is used for a signal feature. Data dimension is $8[$ dimension $]$.

V) Rising voltage level (8[dimension])

The difference of average voltage between the rest state ( 1 second before motion: 3000 [samples]) and activated state ( 1 second after motion: 3000 [sample]) is calculated from each IEMG signal, and it divided by standard deviation. Number of dimension is 8 [dimension].

\section{Methods}

EMG signals were obtained from three male 22-24-year-old subjects total three times in the separate day. Eight electrodes are attached to the subject in the following muscles: pronator teres, spinatior, extensor/flexor carpi ulnaris, flexor/abductor pollicis longus, flexor digitorum superficialis and extensor digitorum. Fig. 1 shows a diagram of the measurement system. During EMG measurement, signal was recorded with a $3 \mathrm{kHz}$-sampling rate. A $60 \mathrm{~Hz}$ ham filter, $10 \mathrm{~Hz}$ Low-pass filter and $100 \mathrm{~Hz}$ High-pass filter were used to remove the noise.

In each measurement, subjects were asked to perform 8 wrist/hand motions in 15 times respectively. The first 10 data were utilized for teaching data, while the last 5 data were for test data.

For the computation of the SVM algorithm, SVM-perf and SVM-multiclass was utilized, which were developed by Cornell university computer science subject [8]. The SVM-multiclass can classify the data into multiple categories, while the SVM-perf did only into two. So, we combined three SVM-perfs to discriminate 8 motions.

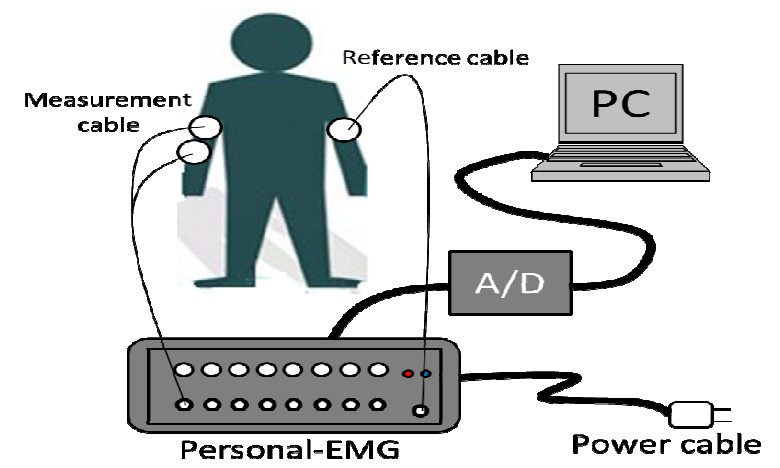

Fig. 1 Measurement system. 


\section{Experiment Result}

Comparison in the composition of data. The discrimination result by SVM-perf and SVM-multiclass are shown in Table. 1 and Table. 2 respectively. The IEMG showed the highest identification rate in (total) average: All subjects showed more than or equal $80 \%$ discrimination rate.

Table1. Result from 3-combined SVM-perf

\begin{tabular}{|c|c|c|c|c|c|c|c|c|c|c|c|c|c|}
\hline & & \multicolumn{2}{|c|}{ Subject A } & \multirow[b]{2}{*}{ Ave } & \multirow[b]{2}{*}{$1 \mathrm{st}$} & \multicolumn{2}{|c|}{ Subject B } & \multirow[b]{2}{*}{ Ave } & \multirow[b]{2}{*}{$1 \mathrm{st}$} & \multicolumn{2}{|c|}{ Subject C } & \multirow[b]{2}{*}{ Ave } & \multirow{2}{*}{\begin{tabular}{|l} 
Total \\
Ave. \\
\end{tabular}} \\
\hline & $1 \mathrm{st}$ & 2nd & $3 r d$ & & & 2nd & $3 r d$ & & & 2nd & $3 r d$ & & \\
\hline Raw & 30 & 15 & 47.5 & 30.8 & 35 & 37.5 & 25 & 32.5 & 40 & 47.5 & 42.5 & 43.3 & 35.6 \\
\hline IEMG & 80 & 72.5 & 87.5 & 80 & 72.5 & 95 & 80 & 82.5 & 100 & 85 & 77.5 & 87.5 & 83. \\
\hline Spectr & 72.5 & 72.5 & 87.5 & 77.5 & 62.5 & 95 & 70 & 75.8 & 82.5 & 87.5 & 75 & 81.7 & 78.3 \\
\hline FFT peak & 30 & 27.5 & 40 & 32.5 & 35 & 35 & 37.5 & 38.8 & 47.5 & 27.5 & 57.5 & 44.2 & 37.5 \\
\hline Vol Level & 62.5 & 82.5 & 95 & 80 & 42.5 & 97.5 & 97.5 & 79.2 & 77.5 & 85 & 87.5 & 83.3 & 80.8 \\
\hline
\end{tabular}

Table2. Result from SVM-multiclass

\begin{tabular}{|c|c|c|c|c|c|c|c|c|c|c|c|c|c|}
\hline & \multicolumn{3}{|c|}{ Subject A } & \multirow[b]{2}{*}{ Ave } & \multirow[b]{2}{*}{$1 \mathrm{st}$} & \multicolumn{2}{|c|}{ Subject B } & \multirow[b]{2}{*}{ Ave } & \multirow[b]{2}{*}{$1 \mathrm{st}$} & \multicolumn{2}{|c|}{ Subject C } & \multirow[b]{2}{*}{ Ave } & \multirow{2}{*}{\begin{tabular}{|l} 
Total \\
Ave. \\
\end{tabular}} \\
\hline & $1 \mathrm{st}$ & 2nd & $3 r d$ & & & 2nd & $3 r d$ & & & 2nd & $3 r d$ & & \\
\hline Raw & 30 & 30 & 50 & 36.7 & 57.5 & 40 & 50 & 49.2 & 50 & 42.5 & 52.5 & 48.3 & 44.7 \\
\hline IEMG & 82.5 & 90 & 95 & 89.2 & 70 & 75 & 95 & 80 & 87.5 & 90 & 80 & 85.8 & 85 \\
\hline Spectr & 72.5 & 80 & 80 & 77.5 & 57.5 & 92.5 & 75 & 75 & 87.5 & 87.5 & 67.5 & 80.8 & 78.3 \\
\hline FFT peak & 45 & 50 & 55 & 50 & 40 & 47.5 & 50 & 45.8 & 32.5 & 47.5 & 52.5 & 44.2 & 46.7 \\
\hline Vol Level & 80 & 77.5 & 87.5 & 81.7 & 37.5 & 75 & 87.5 & 66.7 & 75 & 75 & 87.5 & 79.2 & 75.8 \\
\hline
\end{tabular}

Comparison in the kind of movement. IEMG, power spectrum, and rising voltage level showed around $80 \%$ discrimination rate in previous section. Next, we examine which movement is easy /difficult to discriminate using SVM-perf for the above three signal features showing the higher discrimination rate. Table 3, Table 4, and Table 5 show the comparison between 8 motions for each feature. Overall, the discrimination rate was low in wrist motion. Specially, palmar flexion was resulted in less than $80 \%$ in average of three signal features.

Table 3 Discriminating rates by use of IEMG represented with respect to each movement

\begin{tabular}{|c|c|c|c|c|c|c|c|c|c|c|c|c|c|}
\hline & \multicolumn{3}{|c|}{ Subject A } & & \multicolumn{3}{|c|}{ Subject B } & & \multicolumn{3}{|c|}{ Subject C } & & Total \\
\hline & 1 st & 2nd & $3 \mathrm{rd}$ & Ave & $1 \mathrm{st}$ & 2nd & $3 \mathrm{rd}$ & Ave & $1 \mathrm{st}$ & 2nd & $3 r d$ & Ave & Ave. \\
\hline Pronation & 40 & 80 & 80 & 66.7 & 40 & 100 & 100 & 80 & 100 & 60 & 60 & 73.3 & 73.3 \\
\hline Supination & 100 & 40 & 100 & 80 & 40 & 100 & 60 & 66.7 & 100 & 100 & 80 & 93.3 & 80 \\
\hline Palmar flexion & 40 & 20 & 100 & 53.3 & 80 & 80 & 80 & 80 & 100 & 60 & 80 & 80 & 71.1 \\
\hline Dorsiflexion & 100 & 100 & 60 & 86.7 & 60 & 80 & 80 & 73.3 & 100 & 100 & 80 & 93.3 & 84.4 \\
\hline Thumb flexion & 100 & 60 & 100 & 86.7 & 80 & 100 & 100 & 93.3 & 100 & 80 & 80 & 86.7 & 88.9 \\
\hline Thumb extension & 100 & 100 & 100 & 100 & 80 & 100 & 100 & 93.3 & 100 & 100 & 80 & 93.3 & 95.6 \\
\hline four-finger extention & 80 & 100 & 60 & 80 & 100 & 100 & 40 & 80 & 100 & 100 & 60 & 86.7 & 82.2 \\
\hline four-finger flexion & 80 & 80 & 100 & 86.7 & 100 & 100 & 80 & 93.3 & 100 & 80 & 100 & 93.3 & 91.1 \\
\hline
\end{tabular}

Table 4 Discriminating rates by use of power spectrum represented with respect to each movement

\begin{tabular}{|l|r|r|r|r|r|r|r|r|r|r|r|r|r|r|}
\hline & \multicolumn{4}{|c|}{ Subject A } & \multicolumn{4}{|c|}{ Subject B } & \multicolumn{3}{|c|}{ Subject C } & Total \\
\cline { 2 - 18 } & 1st & 2nd & 3rd & Ave & 1st & 2nd & 3rd & Ave & 1st & 2nd & 3rd & Ave & Ave. \\
\hline Pronation & 60 & 80 & 80 & 73.3 & 80 & 100 & 100 & 86.7 & 100 & 100 & 40 & 80 & 80 \\
\hline Supination & 100 & 40 & 100 & 80 & 40 & 100 & 0 & 46.7 & 40 & 100 & 80 & 73.3 & 66.7 \\
\hline Palmar flexion & 20 & 20 & 100 & 46.7 & 20 & 80 & 20 & 40 & 100 & 100 & 80 & 93.3 & 60 \\
\hline Dorsiflexion & 40 & 100 & 60 & 66.7 & 20 & 80 & 80 & 60 & 60 & 80 & 100 & 80 & 68.9 \\
\hline Thumb flexion & 100 & 60 & 100 & 86.7 & 80 & 100 & 100 & 93.3 & 100 & 80 & 100 & 93.3 & 91.1 \\
\hline Thumb extension & 100 & 100 & 100 & 100 & 80 & 100 & 80 & 86.7 & 100 & 100 & 100 & 100 & 95.6 \\
\hline four-finger extention & 80 & 100 & 60 & 80 & 80 & 100 & 100 & 93.3 & 100 & 100 & 20 & 73.3 & 82.2 \\
\hline four-finger flexion & 80 & 80 & 100 & 86.7 & 100 & 100 & 100 & 100 & 60 & 40 & 80 & 60 & 82.2 \\
\hline
\end{tabular}


Table 5 Discriminating rates by use of rising voltage level represented with respect to each movement

\begin{tabular}{|c|c|c|c|c|c|c|c|c|c|c|c|c|c|}
\hline & \multicolumn{3}{|c|}{ Subject A } & & \multicolumn{3}{|c|}{ Subject B } & & \multicolumn{3}{|c|}{ Subject C } & & Total \\
\hline & 1 st & 2nd & $3 r d$ & Ave & $1 \mathrm{st}$ & 2nd & $3 r d$ & Ave & 1 st & 2nd & $3 \mathrm{rd}$ & Ave & Ave. \\
\hline Pronation & 20 & 80 & 80 & 60 & 0 & 100 & 100 & 66.7 & 80 & 100 & 100 & 93.3 & 73.3 \\
\hline Supination & 80 & 100 & 100 & 93.3 & 20 & 100 & 100 & 73.3 & 80 & 100 & 100 & 93.3 & 86.7 \\
\hline Palmar flexion & 60 & 100 & 100 & 86.7 & 60 & 100 & 100 & 86.7 & 80 & 40 & 40 & 53.3 & 75.6 \\
\hline Dorsif & 80 & 80 & 80 & 66.7 & 40 & 80 & 80 & 66.7 & 60 & 100 & 100 & 86.7 & 77.8 \\
\hline Thumb flexion & 80 & 100 & 100 & 93.3 & 80 & 100 & 100 & 93.3 & 80 & 100 & 100 & 93.3 & 93.3 \\
\hline Thumb extension & 80 & 100 & 100 & 93.3 & 80 & 100 & 100 & 93.3 & 80 & 100 & 100 & 93.3 & 93.3 \\
\hline four-finger extention & 40 & 100 & 100 & 80 & 40 & 100 & 100 & 80 & 80 & 100 & 60 & 80 & 80 \\
\hline four-finger flexion & 60 & 0 & 100 & 73.3 & 20 & 100 & 100 & 73.3 & 80 & 40 & 100 & 73.3 & 66.7 \\
\hline
\end{tabular}

\section{Conclusion}

The IEMG scored $83.3 \%$ discrimination rate. It was the highest of the five signal features; raw signal, integrated signal, the max frequency component, power spectrum, or rising integrated voltage. The nearly $80 \%$ discrimination rate resulted from the power spectrum and the rising voltage level indicates their promising availability as the signal feature of EMG. However, the identification rate differed from day by day: positional deviation from the previous experiment sometimes reduces the voltage difference between the rest and activated state, which prevented the SVM from discriminating hand motions with high discrimination rate. We should find the hot spots of each muscle for each motion of each subject and fixed this position for better motion discriminations, because it will reduce the effect of the positional change of electrodes or the frustration of EMG data in the experiments.

In addition, the result will be improved by shortening the EMG-extraction time period in power spectrum or the rising voltage level since the voltage changes in motions hard to discriminate were small in amplitude and short in time. The rising voltage level results in good discrimination of the wrist motion, while IEMG does in finger motions in comparison to wrist motion. This fact suggests that the usage of several signal features depending on the targeted motions could improve the discrimination rate.

\section{References}

[1] Claudio Castellini, Patrick van der Smagt, suface EMG in advanced hand prosthetics, boil Cybern 100:35-47, 2009.

[2] Satoshi MORITA, Katsunari SHIBATA, Xin-Zin ZHENG, Koji ITO, Human-EMG Prosthetic Hand Interface using Neural Network, IEICE Technical Report MBE99-167, 2000-3.

[3] Yosuke HIRATE, Goro OBINATA, Kazunori KASE, Atushi NAKAYAMA, YoungWoo KIM, Estimation of Hand Motions from Surface Electromyogram by Independent Component Analysis.

[4] Tomoko OTOSHI, Junichi USHIBA, Yutaka TOMITA, Identification of Forearm Movements using Analysis of Electromyographic Signals.

[5] Nello Cristianini, John Shawe-Taylor, An introduction to Support Vector Machines, Cambridge University Press, 2000

[6] Kenzo Akazawa, Huminori Tkizawa, Development of control system and myoelectric signal processor for bio-mimetic prosthetic hand.

[7] Satoshi Ito, Satoshi Ueki, Koji Ishihara, Masayuki Miura, Haruhisa Kawasaki, Yasuhiko Ishigure, Yutaka: Parallel controller construction for a multi-DOF hand rehabilitation equipment, SPIE, ICMIT 2007 Mechatronics, MEMS, and Smart Materials, Vol. 6794, pp.67942B-1-67942B-6, 2007

[8] Thorsten Joachims, Learning to classify text using support vector machines, ISBN 224,2002

[9] http://svmlight.joachims.org/svm_multiclass.html

[10] http://www.cs.cornell.edu/People/tj/svm_light/svm_perf.html 